Research Paper

\title{
Cervical Cancers Manifest a High Rate of Infection by a High-Risk Human Papilloma Virus Subtype but a Very Low Rate of Infection by a Low-Risk Subtype in the Guiyang District of China
}

\author{
Juan Peng1, 2, Yan Yuan,3, Feng Shen¹, Yan Wang ${ }^{1}$, Lichan Chen ${ }^{4}$, D. Joshua Liao ${ }^{5}$, and Yujie Tan ${ }^{1,6 凶}$ \\ 1. Central Laboratory, Guizhou Medical University Hospital, Guiyang, Guizhou 550004, P. R. China; \\ 2. Blood Transfusion Department, TaiHe Hospital Affiliated to HuBei University of Medicine, ShiYan, HuBei 442000, P. R. China; \\ 3. Laboratory Medicine, People's Hospital of Guizhou Province, Guiyang, Guizhou 550002, P. R. China. \\ 4. Hormel Institute, University of Minnesota, Austin, MN 55912, USA. \\ 5. Department of Pathology, Guizhou Medical University Hospital, Guizhou 550004, P.R. China; \\ 6. Laboratory Medicine, Baiyun Hospital Affiliated to Guizhou Medical University Hospital, Guiyang, Guizhou 550014, P. R. China. \\ $\triangle$ Corresponding author: Dr. Yujie Tian, Central Laboratory, Guizhou Medical University Hospital, Guiyang, Guizhou 550001, P. R. China. Tel: \\ 086-0851-86774109 Fax: 086-0851-86774109 E-mail: tanyujie5@163.com
}

(C) Ivyspring International Publisher. This is an open access article distributed under the terms of the Creative Commons Attribution (CC BY-NC) license (https://creativecommons.org/licenses/by-nc/4.0/). See http://ivyspring.com/terms for full terms and conditions.

Received: 2016.08.06; Accepted: 2017.03.31; Published: 2017.05.11

\begin{abstract}
The prevalence of infection by different genotypes of human papillomavirus (HPV) varies among different geographic areas. We studied the prevalence of infection by $21 \mathrm{HPV}$ genotypes in cervical tissue specimens from 4213 women in the Guiyang district, that is located in the southwest of China and is dominated by minor ethnicities of Chinese, and 2074 cases in our cohort had pathological diagnosis available. The overall infection rate was $36.98 \%$. Most $(72.08 \%)$ infectors were positive for only one HPV subtype, with the remaining being cases infected by two or more subtypes. Infections by the HPV subtypes 16, 52 and 58 were the most prevalent, having rates of $34.66 \%, 16.03 \% \%$ and $15.53 \%$, respectively. The most common cervical lesions in HPV infections were genital warts, cervical cancer (CC) and cervical intraepithelial neoplasia (CIN). Age and age at first sexual activity were independent risk factors for HPV infections that in turn cause certain cervical lesions. Intriguingly, while $94.90 \%$ of the CC patients were infected by oncogenically high-risk (HR) HPV subtypes, only $2.75 \%$ and $2.29 \%$ of these patients were infected by oncogenically low-risk (LR) subtypes or other-subtypes with their oncogenicity unclear. The rates of infection by LR-HPVs and other-HPVs were also low, being $4.63 \%$ and $6.76 \%$, respectively, in the patients with CIN that is a precursor lesion of CC, lower than the $8.54 \%$ and $18.20 \%$, respectively, in the women without a cervical lesion. Our data provides an important foundation for prevention, diagnosis and treatment of HPV infection in Guiyang district and suggests that development of vaccines for prevention and treatment of $C C$ in this area should first target the HPV subtypes 16, 52 and 58, but not subtype 18 as for many other places. It deserves study whether infections by certain LR-HPVs and other-HPVs may serve as attenuated live vaccines for prevention of CC.
\end{abstract}

Key words: Human Papillomavirus, Infection, Genotypes, epidemiology investigation.

\section{Introduction}

Cervical cancer (CC) is one of the leading causes of cancer-related mortality among women worldwide. It is estimated that about 500,000 cases occur annually worldwide and half of those patients will die from it [1]. In China, CC ranks as the eighth most frequent cancer type in women and the second most frequent cancer in women of ages 15-44 [2]. Persistent infection of a high risk human papillomavirus (HR-HPV) is essential for the development and progression of high-grade (grades 2 and 3) cervical intraepithelial neoplasia (CIN) that is a main precursor of and a high risk factor for CC [3]. Two HPV vaccines, namely Merck's Gardasil ${ }^{\circledR}$ and GSK's Cervarix ${ }^{\circledR}$, were approved by the Federal Drug Administration of USA in 2006-2007 to protect women from infection by oncogenic HPV subtypes 16 
and 18 and from the lesions caused by the infection. However, prevalence of infection by different HPV subtypes has been reported to vary among different countries or even different areas of the same country $[4,5]$. Therefore, a given vaccine may only be effective in some countries or some areas of a country, and hence vaccines need to be geographically specific to target the subtypes of HPV that are the most prevalent there. The prerequisite for the development of geographically specific vaccines is the availability of information on HPV prevalence. For this reason, in the present study we investigated the prevalence and distribution of HPV subtypes as well as the main risk factors for HPV infections among women in the Guiyang district that is located in the southwest of China.

\section{Materials and Methods}

\section{Specimens and study design}

We conducted a retrospective study with cervical tissue samples from 4213 women of ages 16-75 years who visited Guizhou Medical University Hospital for CC screening during September of 2008 to April of 2012. None of the participants had received any HPV vaccination at the time of sample collection. The sample collection procedure was approved by, and complied with, the institutional ethical committee and the 2013 version of the Declaration of Helsinki of 1975. All participants signed informed consent before enrollment.

Ethnicity, oral contraceptive use, smoking history, age at first sexual intercourse, and the number of sexual partners prior to the enrollment were collected on a voluntary questionnaire filled in by the participants themselves. Histological diagnosis and data of previous cytological screening were retrieved from our hospital databases.

All of the 4213 women underwent cervical biopsy, of which 2074 had enough tissue for pathological diagnosis that was made according to the surgical pathology diagnostic criteria (1999 edition) [6-8]. Of these 2074 women, 67 of whom had virginities, 115 showed genital warts, 1,093 showed cervicitis, 327 had CIN and 235 showed CC, while 237 of the women showed negative cytology and had no any obvious cervical lesion, although they were initially suspected to have cervical diseases macroscopically. These 237 cases were used as the normal controls.

\section{HPV genotyping}

HPV genotyping was performed with the HPV GenoArray test kit (Hybribio biotechnology co., Ltd), which used techniques of both DNA amplification and Hybribio's proprietary flow-through hybridization to simultaneously identify $21 \mathrm{HPV}$ genotypes, including $13 \mathrm{HR}$ or putative-HR HPV subtypes (subtypes 16, 18, 31, 33, 35, 39, 45, 51, 52, 56, 58,59 and 68), 6 low-risk (LR) subtypes (subtypes 6, 11, 42, 43, and 44), and 3 other-HPV subtypes (subtypes 53, 66 and 81) that are unclear for their oncogenicity. The test used a microarray format with a nylon membrane onto which HPV genotype-specific oligonucleotide probes were immobilized. All procedures were carried out following the manufacturer's instructions.

\section{Statistical Analysis}

All the data were analyzed using the SPSS software (version 16.0). HPV infection rates were evaluated with the percentages in the Contingency Table. The $\mathrm{R} \times \mathrm{C}$ chi-square test was used for the differences among groups of HPV infections, with $a \leq 0.05$ considered significant. The cases with complete clinical data were analyzed using univariate analysis, and then the resultant variables were analyzed using multivariate unconditional logistic regression analysis to determine the independent risk factors for HPV infection (Table 1).

\section{Results}

\section{Prevalence and distribution of HPV subtypes}

Of the 4213 cases in our cohort, 1558 (36.98\%) showed positive for at least one of the $21 \mathrm{HPV}$ subtypes studied. HPV subtypes were detected 2181 times in these 1558 women, because many of them were found to be infected by multiple subtypes of HPV. Of the 2181 infections detected, 1596 (73.18\%) were positive for at least one of the $13 \mathrm{HR}-\mathrm{HPV}$ subtypes, $325(14.90 \%)$ were positive for at least one of the 5 LR-HPV subtypes, and $260(11.92 \%)$ were positive for at least one of the 3 other-HPV subtypes. The distribution of different HPV subtypes is shown in table 2. The prevalence of HPV 16 was significantly higher than that of 52 or 58, which was in turn significantly higher than that of any of the rest subtypes $\left(\chi^{2}=106.4, P<0.001\right)$.

Of the 1558 positive specimens, $1123(72.08 \%)$ were positive for only one HPV subtype, 318 (20.41\%) were positive for two HPV subtypes, and 117 (7.51\%) were positive for multiple HPV subtypes, including $73(4.69 \%), 25(1.60 \%), 11(0.71 \%)$ and $8(0.51 \%)$ cases being triple, quadruple, quintuple, and sextuple infections, respectively. These results clearly show that single infection is more common than double and multiple ones $\left(\chi^{2}=113.00, P<0.001\right)$. 
Table 1. Assigned values to variables by logistic regression equation

\begin{tabular}{|c|c|c|}
\hline variables & Anotation & Value and meaning \\
\hline \multirow[t]{2}{*}{ Age(year) } & $\mathrm{x} 1$ & $\leqslant 20=1,>20 \leqslant 25=2,>25 \leqslant 30=3,>30 \leqslant 35=4,>35 \leqslant 40=5$, \\
\hline & & $>41 \leqslant 45=6, \quad>45 \leqslant 50=7,>50 \leqslant 55=8,>55 \leqslant 60=9,>60=10$ \\
\hline \multirow[t]{2}{*}{ Educational } & $\mathrm{x} 2$ & illiteracy=0, primary school=1, middle school=2, \\
\hline & & high school $=3$, university or above $=4$ \\
\hline \multirow[t]{2}{*}{ Occupation } & x3 & unemployed $=0$, peasant $=1$, worker $=2$, businessman $=3$, \\
\hline & & civil servant $=4$ \\
\hline Menopause & $\mathrm{x} 4$ & no $=0$, yes $=1$ \\
\hline Whether to use the lotion & $\mathrm{x} 5$ & no $=0$, yes $=1$ \\
\hline The age of first sex life & $\mathrm{x} 6$ & $\leqslant 20=1, \quad 21-25=2, \geqslant 26=3$ \\
\hline Number of sexual partner & $\mathrm{x} 7$ & $1=1,2=2, \geqslant 3=3$ \\
\hline Times of pregnancy & $\mathrm{x} 8$ & $0=0,1=1,2=2, \geqslant 3=3$ \\
\hline Times of parturition & $\mathrm{x} 9$ & $0=0, \quad 1=1, \geqslant 2=2$ \\
\hline Whether to have cervical lesions & $\mathrm{x} 10$ & no $=0$, yes $=1$ \\
\hline HPV infection & $\mathrm{y}$ & no $=0$, yes $=1$ \\
\hline
\end{tabular}

Table 2. Distribution of HPV subtypes $(n=2181)$

\begin{tabular}{llllll}
\hline Subtype & Cases & $\%$ & Subtype & Cases & $\%$ \\
\hline 6 & 116 & 5.32 & 45 & 18 & 0.83 \\
11 & 152 & 6.97 & 51 & 20 & 0.92 \\
16 & 627 & 28.75 & 52 & 290 & 13.3 \\
18 & 105 & 4.81 & 53 & 87 & 3.99 \\
31 & 72 & 3.3 & 56 & 22 & 1.01 \\
33 & 101 & 4.63 & 58 & 282 & 12.93 \\
35 & 7 & 0.32 & 59 & 17 & 0.78 \\
39 & 43 & 1.97 & 66 & 40 & 1.83 \\
42 & 8 & 0.37 & 68 & 69 & 3.16 \\
43 & 3 & 0.14 & 81 & 97 & 4.45 \\
44 & 5 & 0.23 & & & \\
\hline
\end{tabular}

\section{Relationship between age and HPV infection}

As shown in table 3, we divided the participants into 10 age groups and found that the infection rate in the youngest age group ( $\leq 20$ years) was the highest, followed by the $>55<60$ group and then the $>50<55$ group. The difference between the two groups with the highest infection rates and the differences of these two groups from others were all statistically different $\left(X^{2}=27.99, P<0.01\right)$. However, of all those infected by HR-HPV subtypes, the infection rates of the $>55<60$ and the $\leq 20$ groups were the highest and the second highest $\left(X^{2}=14.86, P<0.01\right)$, respectively. On the other hand, of all those infected by the LR-HPV subtypes, there was a reciprocal change between the age and the infection rate, with the $\leq 20$ group showing the highest infection rate $\left(X^{2}=151.3, P<0.001\right)$. Of those infected by other-HPV subtypes, the youngest group also showed the highest rate $\left(X^{2}=179.86, P<0.001\right)$, followed by the $>55<60$ group and then the $>20<25$ group (Table 3 ).

\section{Distribution of HPV subtypes among patients with different cervical lesions}

The cases with a cervical lesion were divided into 6 groups according to the pathological lesions, and table 4 shows the distribution of HPV subtypes among these groups. Of the 115 patients with genital warts, 87 were HPV positive (75.65\%), including 48 $(55.17 \%$, i.e. $48 / 87)$ cases of single infection and 39 $(44.83 \%$, i.e. $39 / 87)$ cases of double or multiple infections, with HPV6, HPV11 and HPV16 being the most common HPV subtypes. Of the 1093 cervicitis patients, 355 were HPV positive $(32.47 \%)$, including $254(71.54 \%)$ cases with single infection and 101 $(28.45 \%)$ cases with double or multiple infections, with HPV16、 HPV52 and HPV58 being the most common subtypes. Of the 327 CIN patients, 198 were HPV positive $(60.55 \%)$, including $136(68.69 \%)$ cases of single infection and $62(31.31 \%)$ cases of double or multiple infections, with HPV16、HPV58 and HPV52 being the most common subtypes. Of the $235 \mathrm{CC}$ patients, 175 were HPV positive $(74.47 \%)$, including $143(81.71 \%)$ and $32(18.29 \%)$ cases of single infection and double or multiple infections, respectively, with HPV16, HPV58 and HPV18 being the most common subtypes. In most groups of patients with different histological lesions, the single infection rate was much higher than the double or multiple infection rates $(\mathrm{P}<0.01)$, except the group of genital warts that still had $44.83 \%$ of the patients having double or multiple infections.

Table 3. Distribution of HPV subtypes among different age-groups* $(n=4119)$

\begin{tabular}{llllll}
\hline $\begin{array}{l}\text { Age } \\
\text { (year) }\end{array}$ & Cases Total infection & $\begin{array}{l}\text { High-risk } \\
(\mathrm{n}, \%)\end{array}$ & $\begin{array}{l}\text { Low-risk } \\
(\mathrm{n}, \%)\end{array}$ & $\begin{array}{l}\text { Other subtypes } \\
(\mathrm{n}, \%)\end{array}$ \\
\hline$\leq 20$ & 50 & $30(60.00)$ & $19(38.00)$ & $20(40.00)$ & $8(16.00)$ \\
$>20$ & 450 & $176(39.11)$ & $141(31.33)$ & $20(40.00)$ & $30(6.67)$ \\
$>25$ & 714 & $245(34.31)$ & $206(28.85)$ & $60(13.33)$ & $32(4.48)$ \\
$>30$ & 719 & $236(32.82)$ & $206(28.65)$ & $49(6.86)$ & $25(3.48)$ \\
$>35$ & 902 & $342(37.92)$ & $292(32.37)$ & $28(3.89)$ & $47(5.21)$ \\
$>40$ & 571 & $208(36.43)$ & $178(31.17)$ & $55(6.10)$ & $28(4.90)$ \\
$>45$ & 390 & $147(37.69)$ & $127(32.56)$ & $19(3.33)$ & $24(6.15)$ \\
$>50$ & 159 & $66(41.51)$ & $60(37.74)$ & $8(5.03)$ & $7(4.40)$ \\
$>55$ & 71 & $36(50.70)$ & $30(42.25)$ & $5(7.04)$ & $6(8.45)$ \\
$>60$ & 93 & $38(40.86)$ & $35(37.63)$ & $6(6.45)$ & $6(6.45)$ \\
\hline
\end{tabular}

*: Repetitive computation was used for mixed infections by HR-HPV and LR-HPV. 
Table 4. Distribution of HPV subtypes among different cervical-lesion groups $(n=2074)$

\begin{tabular}{lllll}
\hline Lesions & Cases & Infection $(\%)$ & $\chi 2$ & P value \\
\hline Health check & 237 & $19.83(47 / 237)$ & & \\
Vaginitis & 67 & $29.85(20 / 67)$ & 2.667 & 0.141 \\
Genital warts & 115 & $75.65(87 / 115)$ & 62.821 & $0.000^{*}$ \\
Cervicitis & 1093 & $32.47(355 / 1093)$ & 25.563 & 0.000 \\
CIN (I-III) & 327 & $60.55(198 / 327)$ & 34.879 & 0.000 \\
Cervical Cancer & 235 & $74.47(175 / 235)$ & 58.531 & 0.000 \\
Total & 2074 & $42.53(882 / 2074)$ & & \\
\hline
\end{tabular}

Note: Because some cases have multiple infections, infection of each subtype was countered independently.

HPV subtypes were detected 1267 times in a total of 882 specimens that had pathological lesions (table 5 and figure 1), because many samples were detected with multiple subtypes. When compared with those without an obvious lesion and thus assigned to the normal control group, the infection rate by HR-HPVs was the highest, the second highest, and the third highest in the CC, CIN and genital wart groups, respectively $(\mathrm{P}<0.01)$. Among those with LR-HPV infections, the infection rate was the highest in the genital wart group. Among the infections by other-HPVs, the rates were significantly lower in the cases with CC and CIN than in the normal controls $(\mathrm{P}<0.05)$. The most intriguing finding was that cervical cancer patients had a much lower rate of infection by LR-HPVs or other-HPVs, compared with the normal controls or with the patients having other lesions (Table 5). Actually, CIN as a known risk factor for and a precursor lesion of $\mathrm{CC}$ also had lower rates of infections by LR-HPVs and other-HPVs, compared with the normal controls or with the patients having other benign lesions (Table 5).

Figure 1 details the distribution of HPV subtypes in women with different cervical lesions, with the differences among different lesion groups statistically different. Most of those infected by HPV16 were cervicitis patients, followed by CC patients and then CIN patients, while patients with other diseases were much fewer in number $\left(\chi^{2}=25.49, P<0.01\right)$. Most of those infected by HPV52 were cervicitis patients, followed by CIN and then genital wart patients while patients with other diseases were much fewer in number $\left(X^{2}=73.28, P<0.01\right)$. Most of those infected by HPV58 were cervicitis patients, followed by CIN and then CC patients $\left(X^{2}=73.28, P<0.01\right)$. Most of those infected by HPV11 were cervicitis patients, followed by genital warts and CIN patients $\left(\chi^{2}=45.31, P<0.01\right)$, whereas most of those infected by HPV6 were genital wart patients $\left(X^{2}=185.39, P<0.001\right)$. These results suggest that HPVs 16, 52, 58 and 11 might easily cause cervicitis and, meanwhile, HPVs 16 and 58 might be related to $\mathrm{CC}$ development.

Table 5. Infection by different HPV subtypes in patients with different cervical lesions $(n=1267)$

\begin{tabular}{|l|l|l|l|}
\hline Lesion (n) & Infections (\%) & $\chi 2$ & P Value \\
\hline Health check (82) & $73.17(60 / 82) *$ & & \\
\hline & $8.54(7 / 82) * *$ & & \\
\hline & $18.20(15 / 82) * * *$ & & \\
\hline Vaginitis (34) & $73.53(25 / 34) *$ & 0.026 & 1.000 \\
\hline & $14.71(5 / 34) * *$ & 1.705 & 0.276 \\
\hline Genital wart (153) & $11.76(4 / 34) * * *$ & 1.412 & 0.322 \\
\hline & $47.71(73 / 153) *$ & 13.077 & 0.000 \\
\hline & $41.83(64 / 153) * *$ & 28.662 & 0.000 \\
\hline Cervicitis (499) & $10.40(16 / 153) * * *$ & 2.658 & 0.153 \\
\hline & $77.5(387 / 499) *$ & 0.676 & 0.511 \\
\hline & $11.82(59 / 499) * *$ & 0.479 & 0.645 \\
\hline CIN (I -III ) $(281)$ & $10.40(52 / 499) * * *$ & 2.658 & 0.153 \\
\hline & $8.60(249 / 281) *$ & 8.317 & 0.006 \\
\hline Cervical cancer (218) & $64.93(13 / 281) * *$ & 1.229 & 0.407 \\
\hline & $2.75(6 / 218) * *$ & 3.191 & 0.134 \\
\hline & $2.29(5 / 218) * * *$ & 14.222 & 0.000 \\
\hline
\end{tabular}

*: HR-HPV infection, ${ }^{* *}$ : LR-HPV infection, ${ }^{* * *}$ : Other subtype infection

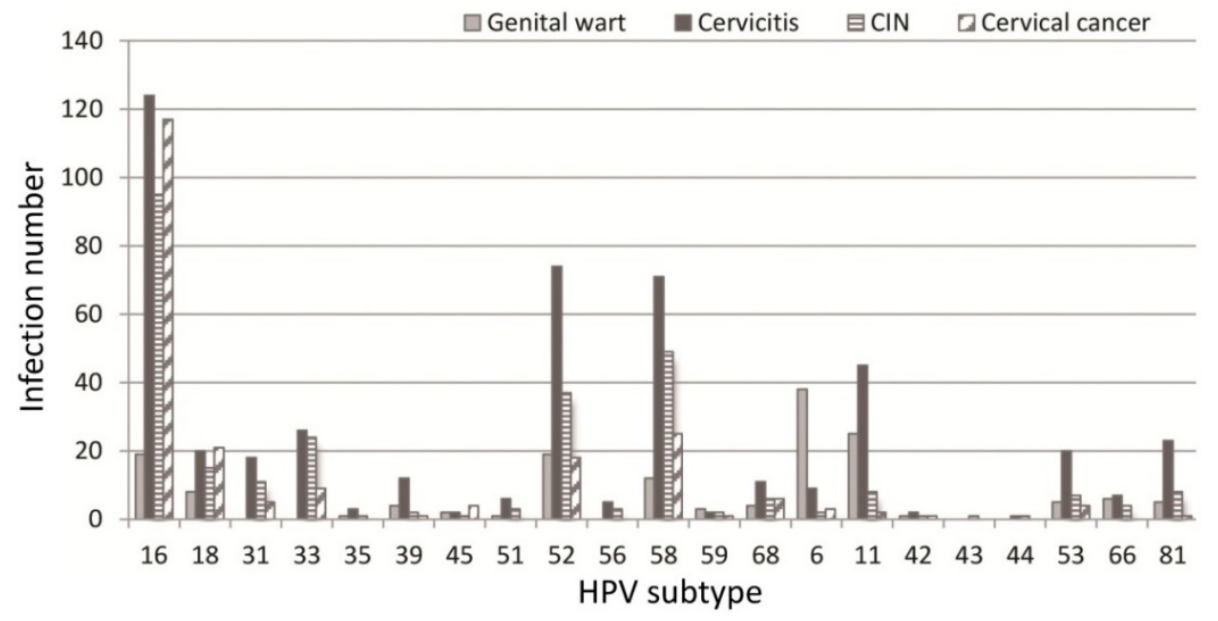

Figure 1. Distribution of 21 HPV genotypes among different cervical lesions. 
Table 6. Relationship between the age of the first sexual activity and HPV infection rate

\begin{tabular}{|l|l|l|l|l|l|}
\hline Age (years) & Cases & Total (\%) & HR-HPV (\%) & LR-HPV (\%) & Other-HPV (\%) \\
\hline$\leq 20$ & 418 & $189(45.22 \%)$ & $161(38.52 \%)$ & $35(8.37 \%)$ & $25(5.98 \%)$ \\
$>20<26$ & 600 & $239(39.83 \%)$ & $201(33.50 \%)$ & $40(6.67 \%)$ & $43(7.17 \%)$ \\
$\geq 26$ & 125 & $42(33.60 \%)$ & $35(28.00 \%)$ & $8(6.40 \%)$ & $5(4.00 \%)$ \\
\hline
\end{tabular}

Table 7. Relationship between the number of sexual partners and HPV infec6tion rate

\begin{tabular}{|l|l|l|l|l|l|}
\hline Partners & Cases & Total (\%) & HR-HPV (\%) & LR-HPV (\%) & Other-HPV (\%) \\
\hline 1 & 825 & $295(35.76 \%)$ & $243(29.45 \%)$ & $52(6.30 \%)$ & $45(5.45 \%)$ \\
\hline 2 & 204 & $107(52.45 \%)$ & $9546.57 \%)$ & $21(10.29 \%)$ & $13(6.37 \%)$ \\
$\geq 3$ & 121 & $70(57.85 \%)$ & $61(50.41 \%)$ & $11(9.10 \%)$ & $14(11.57 \%)$ \\
\hline
\end{tabular}

Table 8. Relationship between the frequency of pregnancy and HPV infection rate

\begin{tabular}{|l|l|l|l|l|l|}
\hline Preg. & Cases & Total $(\%)$ & HR-HPV (\%) & LR-HPV (\%) & Other-HPV (\%) \\
\hline 0 & 66 & $25(37.88 \%)$ & $21(31.82 \%)$ & $5(7.58 \%)$ & $1(1.52 \%)$ \\
\hline 1 & 202 & $77(38.31 \%)$ & $65(32.34 \%)$ & $16(7.96 \%)$ & $10(4.98 \%)$ \\
\hline 2 & 252 & $105(41.83 \%)$ & $83(33.07 \%)$ & $26(10.36 \%)$ & $16(6.37 \%)$ \\
\hline 3 & 383 & $169(44.13 \% *)$ & $144(37.60 \%)$ & $25(6.53 \%)$ & $30(7.83 \%)$ \\
\hline
\end{tabular}

Preg: frequency of pregnancy. *: significantly higher than other groups $(\mathrm{P}<0.05)$.

\section{Relationship between sexual habits and HPV infection}

There were 1143 cases that had sexual activity information available (table 6). These participants were divided into three groups according to their age of the first sexual activity, i.e. the groups $\leq 20,>20<26$, and $\geq 26$ years of age. As shown in table 6, the infection rate descended with the increase in age of the first sexual activity. The difference between any two groups was statistically significant $\left(\chi^{2}=12.43\right.$, $\mathrm{P}<0.05)$. The infection rates of the women who had 1 , 2 , or $\geq 3$ sex partners were $35.76 \%$ (295/895), $52.45 \%$ $(107 / 204)$ and $57.85 \%(70 / 121)$, respectively (table 7$)$, and the difference between any two groups were statistically significant $\left(X^{2}=13.61, P<0.05\right)$.

\section{Relationship between the number of pregnancies or parturitions and HPV Infection}

There were 903 participants with the information on the numbers of pregnancy and parturition available. The HPV infection rate of the women who had experienced $0,1,2$, or $\geq 3$ episodes of pregnancy was $37.88 \%(25 / 66), \quad 38.31 \% \quad(77 / 202), \quad 41.83 \%$ $(105 / 252)$ or $44.13 \%(15 / 66)$, respectively (table 8$)$. The women with $\geq 3$ episodes of pregnancy showed a statistically higher infection rate than those in the other groups $\left(X^{2}=15.430, P<0.05\right)$.

\section{Risk factors for HPV infection}

As shown in table 9, univariate analysis revealed that age, age of first sexual activity, number of sexual partners, number of pregnancies, and cervical lesion type were associated with $\mathrm{HPV}$ infection $(\mathrm{P}<0.05)$. The unconditional logistic regression analysis of the multivariates showed that age, age of first sexual activity and cervical lesion type were associated with HPV infection (Table 10).

Table 9. Results of single risk factor analysis

\begin{tabular}{|c|c|c|c|c|c|}
\hline \multirow{2}{*}{ Factors } & \multirow{2}{*}{ Code } & \multicolumn{2}{|c|}{ Cases } & \multirow{2}{*}{$-x 2$} & \multirow{2}{*}{$P$ value } \\
\hline & & Infected & uninfected & & \\
\hline Age (years) & $\mathrm{x} 1$ & 418 & 485 & 23.721 & 0.003 \\
\hline Education & $x 2$ & 261 & 642 & 2.416 & 0.251 \\
\hline Profession & $\mathrm{x} 3$ & 232 & 671 & 0.521 & 0.841 \\
\hline Postmenopausal & $\mathrm{x} 4$ & 200 & 693 & 0.145 & 0.719 \\
\hline Vaginal wash & $\times 5$ & 203 & 700 & 0.013 & 0.915 \\
\hline First time & $\mathrm{x} 6$ & 350 & 553 & 12.432 & 0.032 \\
\hline Partner \# & $\mathrm{x} 7$ & 384 & 509 & 13.612 & 0.046 \\
\hline Preg \# & $\mathrm{x} 8$ & 376 & 527 & 15.43 & 0.028 \\
\hline Delivery\# & $\mathrm{x} 9$ & 236 & 667 & 11.43 & 0.053 \\
\hline
\end{tabular}

Table 10. Results of logistic regression analysis of risk factors for HPV infection

\begin{tabular}{|l|c|c|c|c|c|}
\hline Variable & $\begin{array}{l}\text { Partial Regression } \\
\text { coefficient }(\mathrm{B})\end{array}$ & Standard error (S.E) & Waldx2 & P Value & OR (95\% confidence interval) \\
\hline Constant term & -1.934 & 0.462 & 17.552 & 0.000 & 0.145 \\
\hline age $(x 1)$ & 0.218 & 0.071 & 9.488 & 0.002 & $1.244(1.083-1.429)$ \\
\hline Initial age $(x 6)$ & -0.751 & 0.198 & 14.436 & 0.000 & $0.472(0.320-0.695)$ \\
\hline Whether lesions $(x 10)$ & 5.42 & 0.317 & 292.023 & 0.000 & $225.940(121.340-420.708)$ \\
\hline
\end{tabular}




\section{Discussion}

\section{Prevalence of infection by different HPV subtype}

There have been over 200 HPV genotypes identified so far, each of which has its own prevalence in different countries or geographic areas, varying with differences in ethnicity, life habit, and social or cultural aspects $[9,10]$. Data from the International Agency of Research on Cancer (IARC) shows that the HPV infection rate in South Africa is $63.9 \%$, in northern Africa is $78.9 \%$, in Central and South America is 65\%, and in Europe and North America is $71.6 \%$ [4]. The HPV infection rate in China has been reported to be much lower, about $14.4 \%$ [11] and $14.9 \%$ [12] in the Wuhan city of Hubei province, although higher figures have been reported in other areas of China, such as $20.80 \%$ in the Shanxi province [13], 33.26\% in the Foshan city of the Guangdong province [14], and $22.6 \%$ in the Hunan province $[15,16]$. The overall HPV infection rate is $36.98 \%$ $(1558 / 4213)$ in our cohort, which is lower than that in Europe, America and Africa but is higher than the above-described figures in other areas of China, probably in part because our participants were hospital visitors. A Meta-analysis showed that the average HPV infection rate among patients with cervical diseases in China or in the north or south of China was $46.5 \%, 55.7 \%$ or $42.1 \%$, respectively [17], which was slightly higher than our data, probably in part because some of our participants visited the hospitals just for a health check and did not have an infection.

Some earlier studies [18], including one from Shanghai [19], reported different distribution patterns of HPV subtypes in different countries. Data from the IARC shows that HPV16 and HPV18 are the most common subtypes worldwide [4]. HPV16, HPV18 and HPV31 have been shown to be the main subtypes in Europe [20]. A Meta-analysis shows that the main infectious subtypes among patients with cervical diseases are HPV16, HPV52, HPV58 and HPV18 [17]. HPV16, HPV52 and HPV58 have been reported to be the main subtypes in the Shanxi province [13], whereas HPV16 and HPV52 are shown to be the main subtypes in Foshan city of Guangdong province [14] and in Wuhan city of Hubei province [13]. These data, including ours, collectively suggest that in China HPV-16 still accounts for the most infections, but infections by HPV-52 and HPV-58 are coming along. A caveat needs to be given that the aforementioned geographical differences may in part be related to the difference in the methods used to detect HPV. In our study, the order of infection prevalence, from the highest to the lowest, is HPV16, HPV52, HPV58,
HPV11, HPV6, HPV18, HPV81, HPV53, HPV33, HPV68 and HPV31. Moreover, the infection rates by HPV16, HPV52 and HPV58 are similar but are higher than that by HPV-18, which belongs to the HR-HPVs. The relatively low prevalence of HPV18 in the Guiyang area of China is unexpected but is of interest, as it suggests that HPV16, HPV52 and HPV58, but not HPV18, should be prioritized when development of vaccines for prevention and treatment of $\mathrm{CC}$ is considered for this part of China.

Different HPV subtypes may cause different pathological lesions, since there have been more than 50 genotypes of HPV that are associated with genital tract lesions [21]. The HR-HPVs that are associated with CIN and CC include HPV16, HPV18, HPV31 and HPV33, whereas the LR-HPVs that are associated with some benign lesions, such as genital warts, include HPV6, HPV11, HPV42 and HPV43. Some other-HPVs have been shown to be associated with CIN or CC in some studies but with benign lesions in some other studies and thus are unclear for their oncogenicity. We observe that the highest HPV infection rates are in the patients with genital warts $(87 / 115$ or $75.65 \%)$ or CC $(75 / 235$ or $74.47 \%)$, all being infected by a single subtype. Moreover, the highest HR-HPV infection rate is seen in the patients with CC $(94.95 \%)$ whereas the highest LR-HPV infection rate is observed in the patients with genital warts $(41.83 \%)$. These results dovetail with a previous study showing that LR-HPV infections mainly cause genital warts, but HR-HPVs cause not only genital warts but also CC [11]. Therefore, genital warts are not always associated with CC. Two intriguing observations in our study are that most participants are infected by only a single HPV subtype and that patients with CC or even just CIN have very low infection rates by LR-HPVs and other-HPVs. In other words, women without a cervical lesion or with a lesion irrelevant to CC have a much higher rate of infection by certain LR-HPVs or other-HPVs, which arouses our suspicion about whether some LR-HPVs or other-HPVs might serve as attenuated live vaccines for $\mathrm{CC}$ prevention.

\section{Risk factors for HPV infection and CC}

Zhao et al. reported that the highest HPV infection rate was in the age group of 17-25 years [22] whereas another study from China showed that the highest HPV infection rate occurred in the age group of 35 44 years [23]. It has also been shown that HPV infections manifest two age-peaks, at age $\leq 25$ years and age $>65$ years [24]. Moreover, most HPV infections among the young patients were transient, and only $5-10 \%$ of the infections would become persistent [24]. We also observe two age-peaks of HPV infections, one at the age of $\leq 20$ years $(60 \%)$ and the 
other at the age of $>55<60$ years $(50.70 \%)$ raising a question as to whether women of these two age groups are either too young or too old to have a stronger innate immunity particularly for protection against HPV infection. Most patients at the first peak got LR-HPV-caused genital warts, whereas most patients with other cervical lesions, including CC and CIN, contributed to the second peak at an older age. Since persistent infection is generally considered a main risk factor for CC and precancerous lesions [25-27], the latter-age peak of infection may be more relevant to $\mathrm{CC}$.

The prevalence of CC has been reported to be significantly higher among the women who had early marriage, early pregnancy and more parturitions [28, 29]. Our data shows that the HPV infection rate of the patients who had their first sexual activity at or before the age of 20 is obviously higher than that in other age groups, generally in line with the opinion that having sexual intercourse at an earlier age might increase the risk for CC. Our multivariate logistic regression analysis shows that age, age at the first sexual activity and histological type of cervical lesion are associated with CC. It is logical to conclude that age and age at first sexual activity are likely to be risk factors for the infections that in turn cause certain histological types of cervical lesion.

Taken together, for the first time, we provide data of the prevalence and distribution of HPV subtypes in women in the Guiyang area of China, which can be an important foundation for diagnosis, treatment and prevention of HPV infection in this area. Our cohort is one of the largest in terms of the number of cases and the number of HPV subtypes documented in the literature. The most prevalence of subtypes in this area of China, which is dominated by minor ethnicities of Chinese, is HPVs 16, 52 and 58, but not HPV 18 that is common in many other parts of the world. While most women were infected by only a single HPV subtype, most CC patients have been infected by a HR-HPV subtype but rarely by an LR-HPV or other-HPV, which collectively raises a possibility of whether certain LR-HPVs and other-HPVs may serve as attenuated live vaccines.

\section{Acknowledgments}

We thank Wei Wang for specimen collection and YingJie Yang for the excellent technical assistance. We would also like to thank Dr. Fred Bogott at the Austin Medical Center, Austin of Minnesota, USA, for his excellent English editing of the manuscript. This work was supported by the Science and Technology Bureau of Guiyang City (Grant No. 31360544 to YJ Tan) and by the
Department of Science and Technology of Guizhou Province (No.2011037 to YJ Tan).

\section{Competing Interests}

The authors have declared that no competing interest exists.

\section{References}

1. Baleriola C, Millar D, Melki J, et al. Comparison of a novel HPV test with the Hybrid Capture II (hcII) and a reference PCR method shows high specificity and positive predictive value for 13 high-risk human papillomavirus infections. J Clin Virol. 2008; 42: 22-6.

2. Ferlay J, Shin HR, Bray F, et al. Cancer incidence and mortality worldwide: IARC CancerBase. International Agency for Research on Cancer. 2012; 10: 1.

3. Pierce Campbell CM, Menezes LJ, Paskett ED, et al. Prevention of invasive cervical cancer in the United States: past, present, and future. Cancer Epidemiol Biomarkers Prev. 2012; 21: 1402-8.

4. Munoz N, Bosch FX, De SS, et al. Epidemiologic classification of human papillomavirus types associated with cervical cancer. N Engl J Med. 2003; 348: 518-27.

5. Wheeler CM, Hunt WC, Joste NE, et al. Human papillomavirus genotype distributions: implications for vaccination and cancer screening in the United States. J Natl Cancer Inst. 2009; 101: 475-87.

6. Lin ZQ. Cervical neoplasm. In: Xie X and Gou WL, ed. Obstetrics and Gynecology. Beijing: People's Medical Publishing House. 2013: 301-9.

7. Xie FX. Inflammation of hte vulva and vagina. In: Xie X, Gou WL eds. Obstetrics and Gynecology [8], 245-252. 1-21-2013. Beijing: People's Publishing House.

8. Xue FX. Cervical inflammation. In: Xie X, Gou WL, eds. Obstetrics and Gynecology. Beijing: People's Medical Publishing House. 2013: 457-72.

9. Dong XJ., Zhang GR., Wang SB., et al. Molecular epidemiological study on sex transmitting HPV16, 18 and the screening of a new HPV strain. Chinese Journal of Infectious Diseases. 2001; 19: 281-4.

10. Koshiol J, Lindsay L, Pimenta JM, et al. Persistent human papillomavirus infection and cervical neoplasia: a systematic review and meta-analysis. Am J Epidemiol. 2008; 168: 123-37.

11. Bao YP, Li N, Smith JS, et al. Human papillomavirus type distribution in women from Asia: a meta-analysis. Int J Gynecol Cancer. 2008; 18: 71-9.

12. Zhou J, Liu Q. Prevalence and type distribution of human papillomavirus infection in women of Wuhan city. Lab Med Clinic. 2010; 7: 1155-6.

13. Rong $\mathrm{S}, \mathrm{Chen} \mathrm{W}, \mathrm{Wu} \mathrm{L}$, et al. [Analysis of risk factors for cervical cancer in Xiangyuan County, Shanxi Province]. Zhonghua Yu Fang Yi Xue Za Zhi. 2002; 36: $41-3$.

14. Song CL, Fan JH, Chen SF, et al. Analysis of subtyes with human papillomavirus infection in Foshan area. Chin Maternity Child Health Study. 2010; 21: 332-5.

15. Liu $\mathrm{W}, \mathrm{Wu}$ EQ, $\mathrm{Yu} \mathrm{XH}$, et al. Detection of human papillomavirus genotypes associated with mucopurulent cervicitis and cervical cancer in Changchun, China. Int J Gynaecol Obstet. 2013; 120: 124-6.

16. Li H, Zhang J, Chen Z, et al. Prevalence of human papillomavirus genotypes among women in Hunan province, China. Eur J Obstet Gynecol Reprod Biol. 2013; 170: 202-5.

17. Zhang DH, Lin MS. Meta-analysis of human papillomavirus (HPV) prevalence and type distribution in cervical lesions in Chinese population. Chin General Practice. 2010; 13: 1287-90.

18. Ghaffari SR, Sabokbar T, Mollahajian H, et al. Prevalence of human papillomavirus genotypes in women with normal and abnormal cervical cytology in Iran. Asian Pac J Cancer Prev. 2006; 7: 529-32.

19. Huang S, Afonina I, Miller BA, et al. Human papillomavirus types 52 and 58 are prevalent in cervical cancers from Chinese women. Int J Cancer. 1997; 70: 408-11.

20. Carlson JA, Cribier B, Nuovo G, et al. Epidermodysplasia verruciformis-associated and genital-mucosal high-risk human papillomavirus DNA are prevalent in nevus sebaceus of Jadassohn. J Am Acad Dermatol. 2008; 59: 279-94.

21. Lee EH, Um TH, Chi HS, et al. Prevalence and distribution of human papillomavirus infection in Korean women as determined by restriction fragment mass polymorphism assay. J Korean Med Sci. 2012; 27: 1091-7.

22. Zhao FH, Zhu FC, et al. Baseline prevalence and type distribution of human papillomavirus in healthy Chinese women aged 18-25 years enrolled in a clinical trial. Int J Cancer. 2014; 135: 2604-11.

23. Bao $\mathrm{YP}, \mathrm{Li} \mathrm{N}$, Wang $\mathrm{H}$, et al. [Study on the distribution of human papillomavirus types in cervix among Chinese women: a meta-analysis]. Zhonghua Liu Xing Bing Xue Za Zhi. 2007; 28: 941-6.

24. Guardado-Estrada M, Juarez-Torres E, Roman-Bassaure E, et al. The distribution of high-risk human papillomaviruses is different in young and old patients with cervical cancer. PLoS One. 2014; 9: e109406.

25. Jia Y. Clinical value of human papillomavirus genotype distribution in HPV infection. Acta Academiae Medicinae Militaris Tertiae. 2009; 31: 2387-9. 
26. Grozdanov P, Zlatkov V, Ganchev G, et al. HPV prevalence and type distribution in women with normal or abnormal Pap smear in Bulgaria. J Med Virol. 2014; 86: 1905-10.

27. Cuschieri KS, Cubie HA, Whitley MW, et al. Persistent high risk HPV infection associated with development of cervical neoplasia in a prospective population study. J Clin Pathol. 2005; 58: 946-50.

28. Coser J, da Rocha BT, Simon D, et al. Prevalence and genotypic diversity of cervical human papillomavirus infection among women from an urban center in Brazil. Genet Mol Res. 2013; 12: 4276-85.

29. Coser J, Boeira TR, Wolf JM, et al. Cervical human papillomavirus infection and persistence: a clinic-based study in the countryside from South Brazil. Braz J Infect Dis. 2016; 20: 61-8. 